\title{
Sistema auxiliar de bombeamento de solução nutritiva em cultivos hidropônicos de hortaliças
}

\author{
João Bosco C da Silva'; Carlos Arthur N Machado'; Juliana G Monteiro² \\ ${ }^{1}$ Embrapa Hortaliças, C. postal 218, 70359-970 Brasília-DF; ${ }^{2}$ UEG, Campus de Ipameri, Rodov. GO 330, km 241 s/n, Ipameri-GO; \\ jbosco@enph.embrapa.br
}

\section{RESUMO}

O sistema de hidroponia mais utilizado consiste em cultivar plantas em calhas ou outros contentores por onde circula a solução nutritiva de forma intermitente. Este processo exige disponibilidade permanente de energia elétrica para o bombeamento de solução nutritiva, podendo ocorrer grandes perdas no caso de falhas prolongadas no fornecimento elétrico. Foram avaliados dois sistemas auxiliares de bombeamento. Em um deles, a circulação da solução é feita por força de ar comprimido que, por controle de nível com bóia elétrica e acionamento de válvulas pneumáticas, permite realizar diversos ciclos de circulação da solução nutritiva com o ar acumulado no cilindro do compressor. O outro sistema consiste em um conjunto de motobombas que funcionam com energia de 12 volts, acumulada em baterias abastecidas por carregador. O sistema para testes foi composto por um compressor de $1 \mathrm{cv}$ com cilindro de ar de aproximadamente $45 \mathrm{~L}$ e dois reservatórios de solução, sendo o inferior com capacidade de $60 \mathrm{~L}$, que permite a pressurização. Especificamente para o compressor utilizado obteve-se em média, quatro ciclos de transferência de solução por cada carga do compressor cheio e desligado. $\mathrm{O}$ volume total de solução movimentada por cada carga do compressor foi de $200 \mathrm{~L}$, o que corresponde à movimentação de aproximadamente $5 \mathrm{~L}$ de solução por litro de ar comprimido. O outro sistema consiste de três motobombas de 12 volts, modelo 500 gph com capacidade de recalque de $1890 \mathrm{~L} \mathrm{~h}^{-1}$ e consumo de 2,5 ampér $\mathrm{h}^{-1}$, acionadas por uma bateria automotiva. O conjunto funcionou, em média, durante 3 horas e 20 minutos para cada carga da bateria. $\mathrm{O}$ volume de solução movimentado foi de aproximadamente $500 \mathrm{~L}$ $\mathrm{h}^{-1}$. Com os parâmetros obtidos é possível dimensionar os sistemas de bombeamento auxiliar de acordo com o volume de solução a ser movimentado e com o intervalo de segurança que for conveniente, pois existem no mercado inúmeros modelos de compressores de ar, baterias automotivas de várias capacidades de carga e vários modelos de motobomba de 12 volts.

Palavras-chave: Hidroponia, bombeamento de segurança, circulação de solução.

\begin{abstract}
Auxiliary pumping of nutrient solution for hydroponic culture of vegetables

The most usual hydroponics system consists of plant cultivation into gutter pipe or other containers through which the nutrient solution circulates intermittently. This system requires continuous electricity supply for pumping nutrient solution, so it is highly vulnerable to prolonged failures in the electricity supply. Two auxiliary systems were evaluated for pumping. In one, the solution movement is promoted by compressed air. The electric buoys and pneumatics valves controllers perform several cycles of nutrient solution, using the air accumulated into the cylinder's compressor. The other system consists of a set of 12-volt pumps fed by batteries which are supplied by a charger. The tested system had a compressor with a motor of one hp, a cylinder of $45 \mathrm{~L}$, and two solution tanks. One tank had 60 L, which allows pressurization. Specifically for the used compressor, it was obtained, on average, four cycles of solution transference for each full cylinder of the compressor, without electricity. The total volume of solution transference for each cylinder was 200 L, which corresponds to the movement of approximately $5 \mathrm{~L}$ of solution per liter of compressed air. The other system consists of three 12-volt pumps model $500 \mathrm{gph}$ with capacity for $1890 \mathrm{~L} \mathrm{~h}^{-1}$ of liquid transference and consumption of $2.5 \mathrm{amps} \mathrm{h}^{-1}$, supplied by an automotive battery. The set worked, on average, for 3 hours and 20 minutes for each battery charge. The volume of pumped solution was approximately $500 \mathrm{~L} \mathrm{~h}^{-1}$. With these parameters it is possible to make projects of systems for auxiliary pumping according to the volume of solution to be pumped and the convenient intervals, since there are many models of air compressors, automotive batteries, chargers and 12-volts pumps.
\end{abstract}

Keywords: Hydroponics, safety pumping, circulation of solution.

(Recebido para publicação em 19 de março de 2009; aceito em 27 de agosto de 2010) (Received on March 19, 2009; accepted on August 27, 2010)

$\mathrm{D}$ iversas espécies olerícolas são cultivadas no sistema hidropônico. O sistema mais comum consiste no cultivo das plantas em calhas ou outros contentores, com fornecimento da solução nutritiva de forma intermitente. A solução nutritiva é inicialmente recalcada para um depósito superior, localizado acima do nível das calhas de cultivo. A solução é conduzida por gravidade, passa pelas calhas e é recolhida em um depósito inferior de onde é novamente recalcada para o depósito superior (Castelane \& Araújo, 1995; Furlani, 1998).

O sistema de circulação de solução nutritiva é geralmente acionado por energia elétrica. As falhas prolongadas no fornecimento de energia podem cau- sar grande prejuízo, devido ao ressecamento do sistema radicular das plantas. Este é um fator limitante, principalmente em locais distantes dos centros urbanos, onde as falhas são mais frequentes e mais prolongadas, devido às dificuldades no atendimento das equipes de manutenção.

$\mathrm{O}$ uso de geradores elétricos es- 
tacionários é a opção mais utilizada para garantir a continuidade do bombeamento da solução nutritiva, porém estes equipamentos são relativamente caros, especialmente quando dotados de sistemas automatizados de partida e desligamento.

Outra opção poderia ser a utilização de painéis de captura de radiação solar para acumular energia em baterias de 12 $\mathrm{V}$ e estas acionarem bombas (Combrink \& Harms, 2001). Contudo, os painéis geram apenas cerca de $18 \mathrm{w} \mathrm{h}^{-1}$, durante o período de exposição, o que é muito pouco se considerarmos que um motor elétrico de $1 \mathrm{cv}$ consome $750 \mathrm{w} \mathrm{h}^{-1}$.

Neste trabalho são apresentadas duas alternativas de sistemas paralelos ao bombeamento convencional, acionados e revertidos automaticamente durante os intervalos de interrupção de energia elétrica.

\section{MATERIAL E MÉTODOS}

Os dois sistemas propostos foram instalados na Embrapa Hortaliças, de forma não associada ao sistema de cultivo, visando avaliar o funcionamento e estabelecer os parâmetros iniciais para utilização destes sistemas.

Função dos sistemas - As instalações propostas funcionam de forma paralela ao sistema de bombeamento convencional, sendo dotados de mecanismos automáticos de acionamento e reversão entre o sistema paralelo e o convencional, todas as vezes que ocorrer a interrupção e o restabelecimento no fornecimento de energia elétrica.

Sistema de 12 volts - O sistema consiste em utilizar baterias automotivas para mover uma ou mais motobombas auxiliares. $O$ conjunto avaliado consiste de três motobombas que funcionam com energia de 12 volts, acumulada em baterias abastecidas por carregador durante os períodos de fornecimento de energia elétrica. Possui ainda um relê temporizador, chave contadora e chave de nível, que automatizam o funcionamento e a reversão para o bombeamento convencional quando o abastecimento for restabelecido.

Funcionamento - $\mathrm{Na}$ figura 1 é apresentado o esquema de instalação do

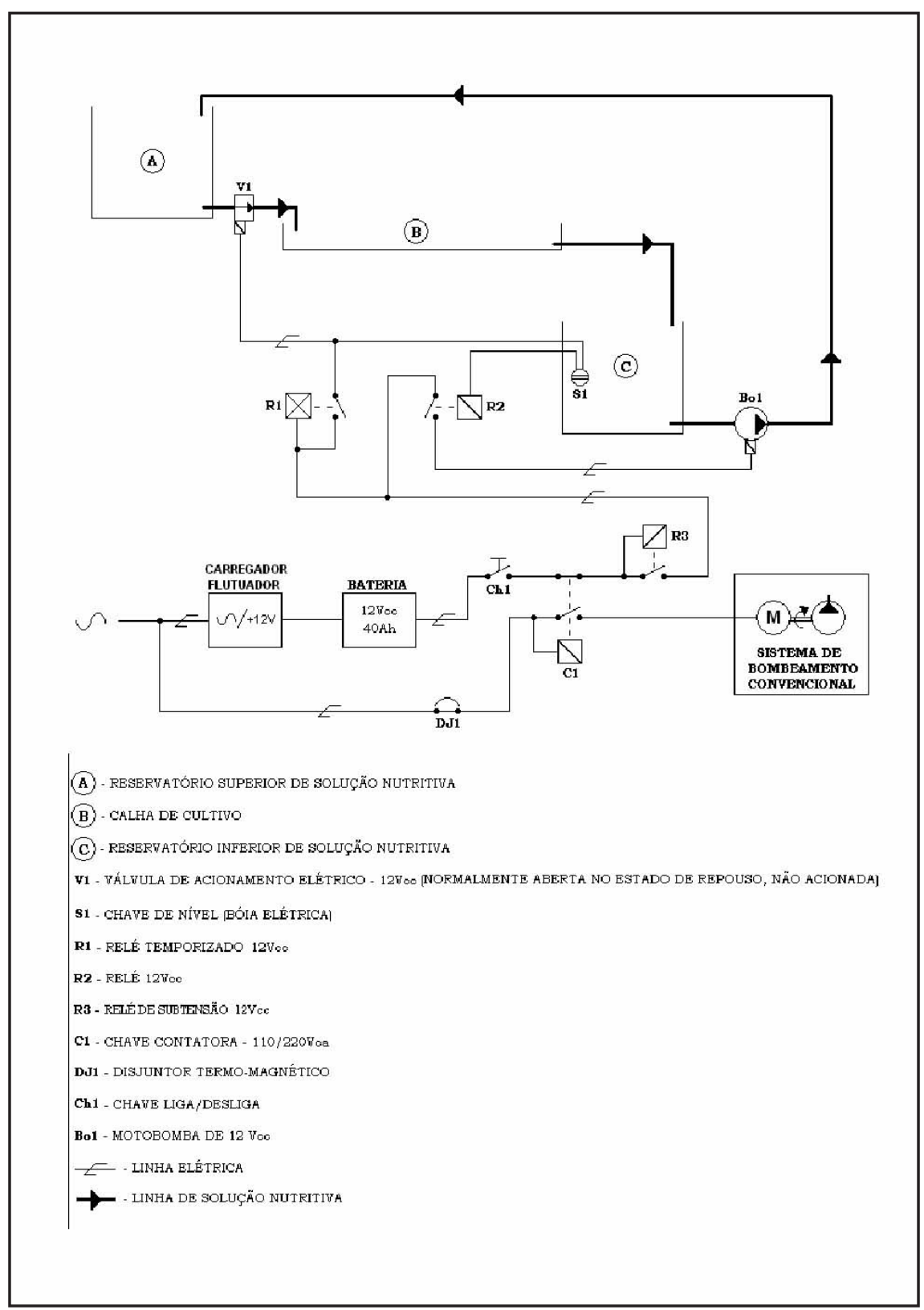

Figura 1. Sistema de bombeamento auxiliar por motobombas de 12 volts (auxiliary pumping system through 12-volt pumps). Brasília, Embrapa Hortaliças, 2008.

sistema e na figura 3, o sistema instalado. Enquanto a rede de energia elétrica convencional estiver presente, a chave contactora (C1) permanece energizada, abrindo o circuito alternativo de 12 volts e mantendo o circuito de bombeamento convencional funcionando. Durante este tempo, a bateria de 12 volts é carregada e mantida em carga máxima, por meio de um Carregador Flutuador, que permite ficar ligado na rede elétrica por tempo indeterminado.

$\mathrm{Na}$ falta de energia elétrica da rede, a chave contactora (C1) abre o circuito de bombeamento convencional e fecha o circuito alternativo de 12 volts. A tensão de 12 volts fecha o contato do relé de subtensão (R3), que dispara o temporizador (R1), de acordo com tempo regulado, fechando o contato do relé (R2) que aciona as motobombas durante os intervalos programados. O líquido (solução nutritiva) contido no reservatório inferior (C), localizado abaixo do nível da calha de cultivo é recalcado para a extremidade superior da calha ou para outro reservatório (A).

De acordo com o tempo programado, é acionada a válvula solenóide (V1), através do temporizador (R1), fechando 
ou abrindo a saída de solução nutritiva do reservatório (A). A chave de nível (S1) instalada no reservatório inferior (C) aciona ou desativa o funcionamento das bombas de acordo com o nível de líquido contido no reservatório. De acordo com o tempo programado, o relé temporizado abre ou fecha o seu contato e a solenóide (V1), permitindo ou não que a solução nutritiva armazenada no reservatório superior escoe pela calha de cultivo. Estes ciclos irão se repetir enquanto a carga da bateria permitir, pois quando a mesma abaixar para 10,5 volts, o relé de subtensão (R3) abrirá o seu contato, desligando o circuito alternativo, evitando, portanto o dano aos motores das bombas.

Sistema com ar comprimido - $\mathrm{Na}$ figura 2 é apresentado o esquema da instalação e na figura 4, o sistema instalado. A circulação da solução é feita por força de ar comprimido que, ao ser aplicado em um depósito vedado, força a saída da solução contida dentro dele e a sua transferência para outro depósito localizado acima das calhas. $\mathrm{O}$ controle de nível com bóia elétrica e o acionamento de válvulas pneumáticas permitem realizar diversos ciclos de circulação da solução nutritiva com o ar acumulado no cilindro do compressor durante o período de fornecimento de energia elétrica.

Funcionamento - Ao se aplicar o ar comprimido no reservatório vedado (C) (Figura 2), a solução nele contida sai pela tubulação instalada no fundo e se transfere para o reservatório elevado (A). Ao final do processo de transferência da solução, a chave de nível (S1) instalada dentro do reservatório inferior, interrompe o acionamento da válvula (V3) que corta o fluxo de ar, ao abrir o circuito de $12 \mathrm{v}$ que comanda a válvula solenóide controladora de ar (V3). No mesmo instante, devido à ausência de pressão na linha pneumática, ocorre também a abertura das válvulas de água (V1 e V5) e do suspiro (V2), permitindo o retorno da solução nutritiva do reservatório superior para o inferior, passando pela calha (B) onde são cultivadas as plantas.

Quando o nível da solução transferida para o reservatório inferior atinge o máximo, a chave de nível (S1) fecha o circuito de $12 \mathrm{v}$ que aciona novamente

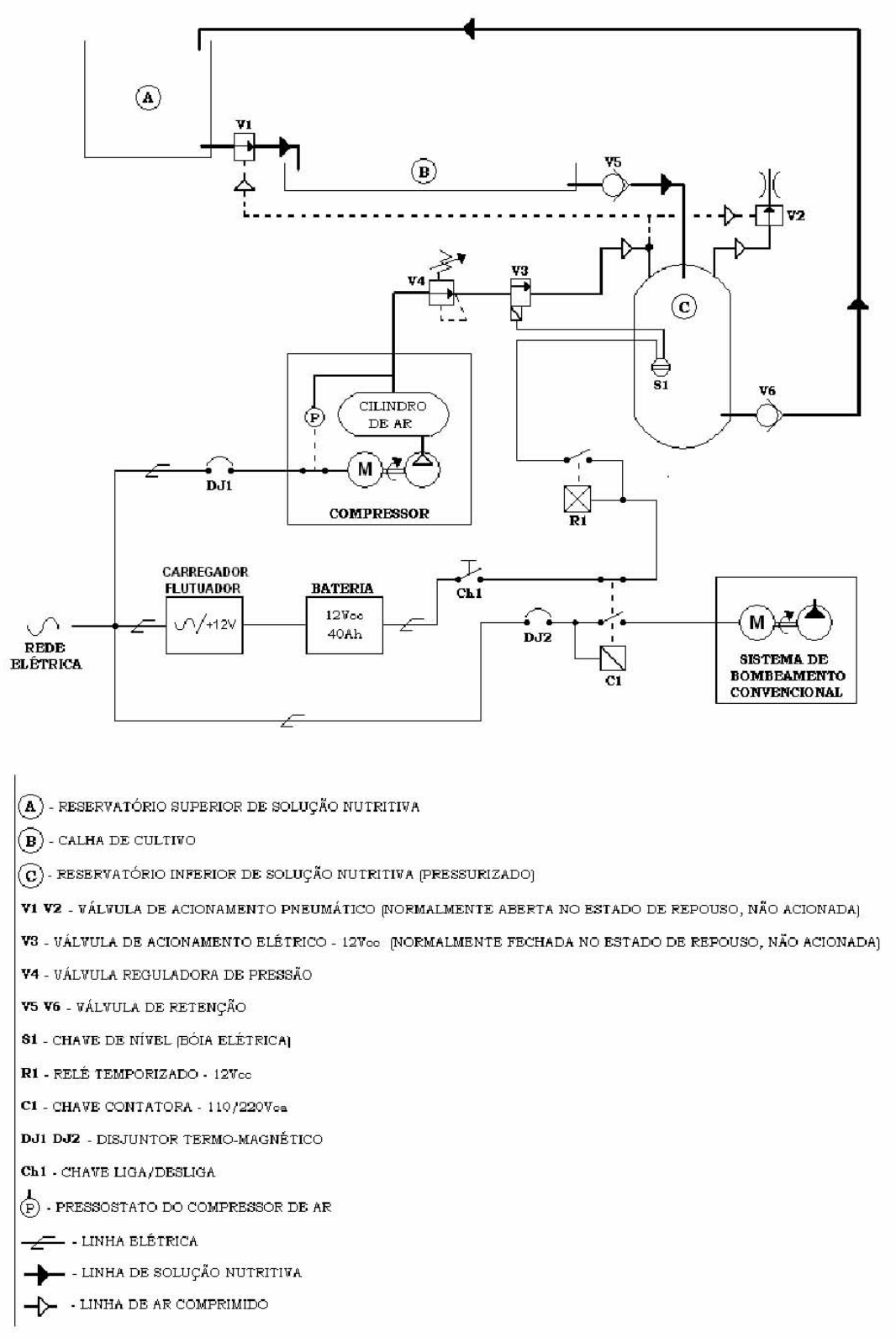

Figura 2. Sistema de bombeamento auxiliar por meio de ar comprimido (auxiliary pumping system through compressed air). Brasília, Embrapa Hortaliças, 2008.

o sistema pneumático, abrindo a válvula de ar comprimido (V3) que injeta pressão no reservatório inferior, fazendo a transferência do líquido para o reservatório superior. No mesmo instante, a válvula de água (V1), instalada no cano de saída do reservatório superior, é fechada, interrompendo o fluxo da solução pela calha. A válvula do suspiro (V2) também é fechada, para permitir a pressurização do reservatório. A válvula de retenção (V2), instalada no cano de entrada do reservatório inferior, não permite o retorno da solução nutritiva e nem a saída do ar.
O intervalo entre ciclos de circulação da solução nutritiva é estabelecido por meio de um temporizador (R1) que controla a energia que aciona a válvula (V3) e esta controla o fluxo de ar comprimido de todo o sistema.

O ciclo de circulação se repete até que a pressão do ar contido no depósito do compressor não seja mais suficiente para acionar as válvulas, principalmente a (V3), que controla o fluxo de ar.

A reversão para o sistema convencional de bombeamento se dá quando a energia elétrica aciona o relê (R1) que abre o circuito de 12 volts, interrompen- 


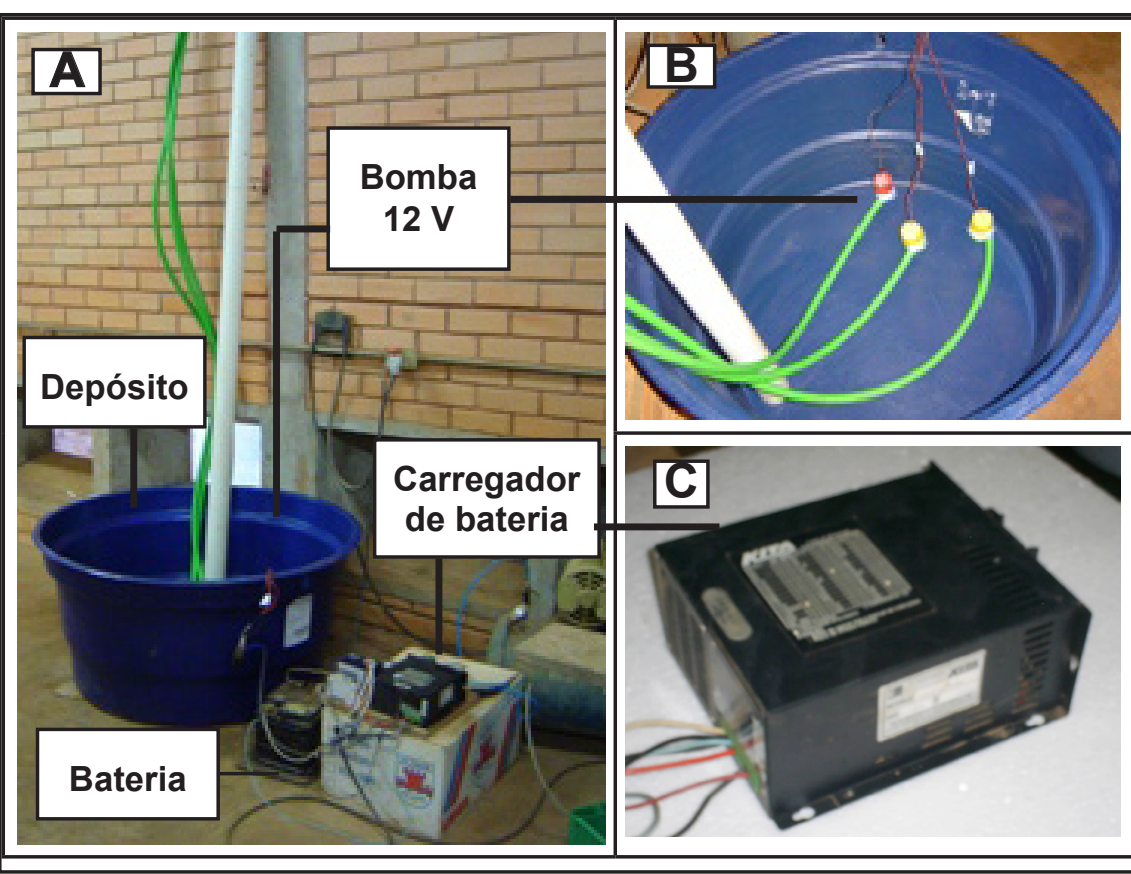

Figura 3. Sistema auxiliar de bombeamento, utilizando bombas de 12 volts (auxiliary pumping system using 12-volt pumps). Brasília, Embrapa Hortaliças, 2008.

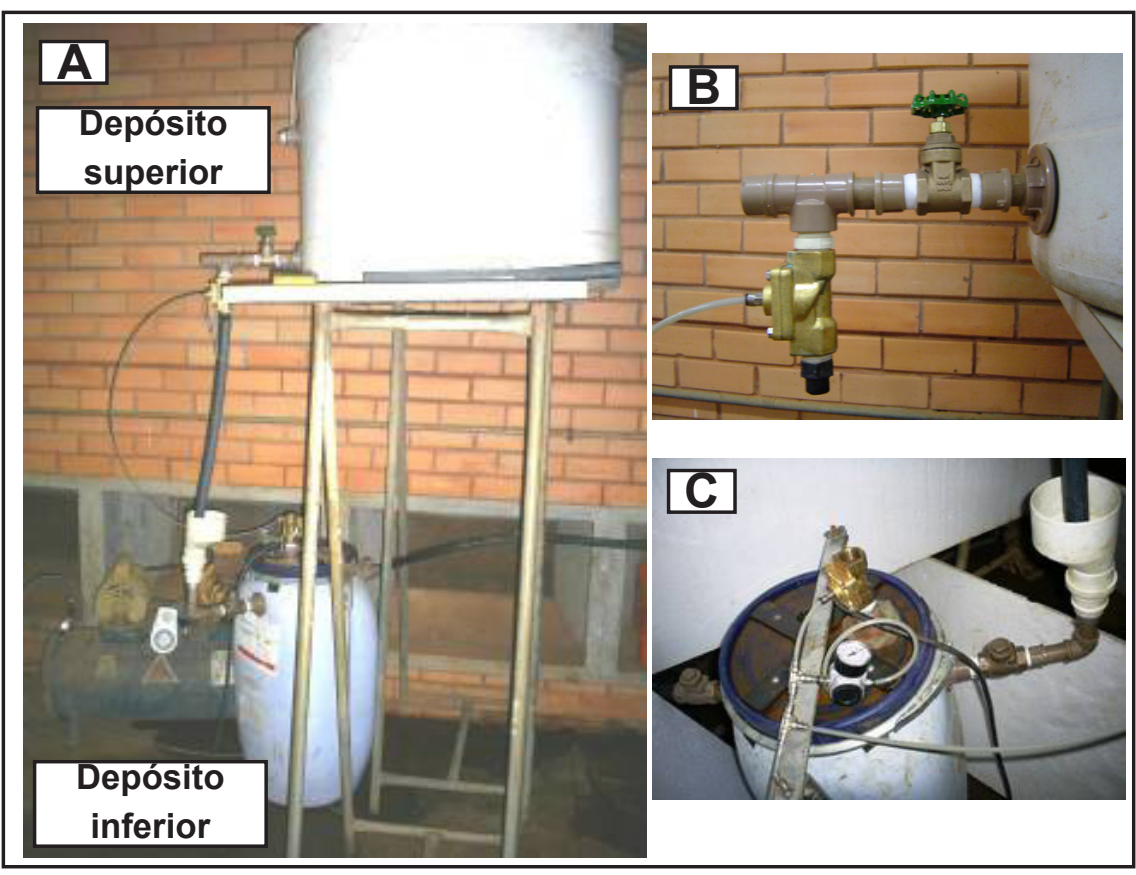

Figura 4. Sistema auxiliar de bombeamento, utilizando ar comprimido. A - protótipo experimental,. B - válvula solenóide, C - sistema de controle. (auxiliary pumping system through compressed air. A - experimental prototype, B - solenoid valve, C - controlers) Brasília, Embrapa Hortaliças, 2008.

do o funcionamento da válvula (V3), que por sua vez, interrompe o fluxo de ar comprimido.

Enquanto houver o fornecimento de energia elétrica o compressor se recarrega e mantém o reservatório de ar comprimido na pressão máxima, de acordo com a faixa de pressão estabelecida pelo contendo cerca de $15 \mathrm{~cm}$ de lâmina de água, apenas o suficiente para manter as bombas submersas. As bombas foram conectadas a tubulações (mangueiras $3 / 4$ de polegada) com $3 \mathrm{~m}$ de comprimento, posicionadas para as alturas de recalque de $1,5 \mathrm{~m}$ e $2,5 \mathrm{~m}$. Foram realizadas três medições de vazão de cada bomba, durante dez minutos, com o temporizador regulado para cinco minutos de funcionamento e trinta segundos de interrupção. As medições foram feitas com uma bateria nova, de 45 ampér e completamente carregada e também com uma bateria em final de vida útil.

Para o sistema de ar comprimido, o protótipo (figura 4) foi composto por um compressor com motor de $1 \mathrm{cv}$, com cilindro de ar de aproximadamente $45 \mathrm{~L}$ e o consumo aproximado de 1,2 Kwh. Dois reservatórios de solução, sendo o inferior com capacidade de $60 \mathrm{~L}$, que permite a pressurização, e outro, com capacidade para $250 \mathrm{~L}$, que foi instalado na altura de $3 \mathrm{~m}$. Os ciclos de circulação da solução nutritiva foram controlados por válvulas pneumáticas, controladoras de pressão e bóias elétricas.

\section{RESULTADOS E DISCUSSÃO}

Com a bateria nova, as três bombas de 500 gph funcionaram durante seis horas e vinte minutos. Depois de três horas de funcionamento, a vazão era de $564 \mathrm{~L} \mathrm{~h}^{-1}$, tendo um pequeno declínio de vazão ao longo do tempo. Com a bateria usada, a primeira medição da vazão, medida após a primeira hora de funcionamento foi de $536 \mathrm{~L} \mathrm{~h}^{-1}$. Após duas horas e trinta minutos a vazão estava em $360 \mathrm{~L} \mathrm{~h}^{-1} \mathrm{e}$ a bateria se esgotou com três horas de funcionamento, apresentando a vazão de $270 \mathrm{~L} \mathrm{~h}^{-1}$. Efetuada a regressão linear dos dados obteve-se a equação: $\mathrm{Y}\left(\mathrm{L} \mathrm{h}^{-1}\right)=-1,867 \mathrm{X}(\mathrm{min})+631,31\left(\mathrm{R}^{2}\right.$ $=0,8854)$.

Embora as três bombas sejam da mesma especificação de vazão (500 gph) e os fabricantes não citem nenhuma outra característica que possam diferenciá-las, uma delas apresentou o desempenho inferior (bomba 3 na figura 5). As outras bombas apresentaram desempenho similar entre si, mesmo sendo de fabricantes diferentes. 


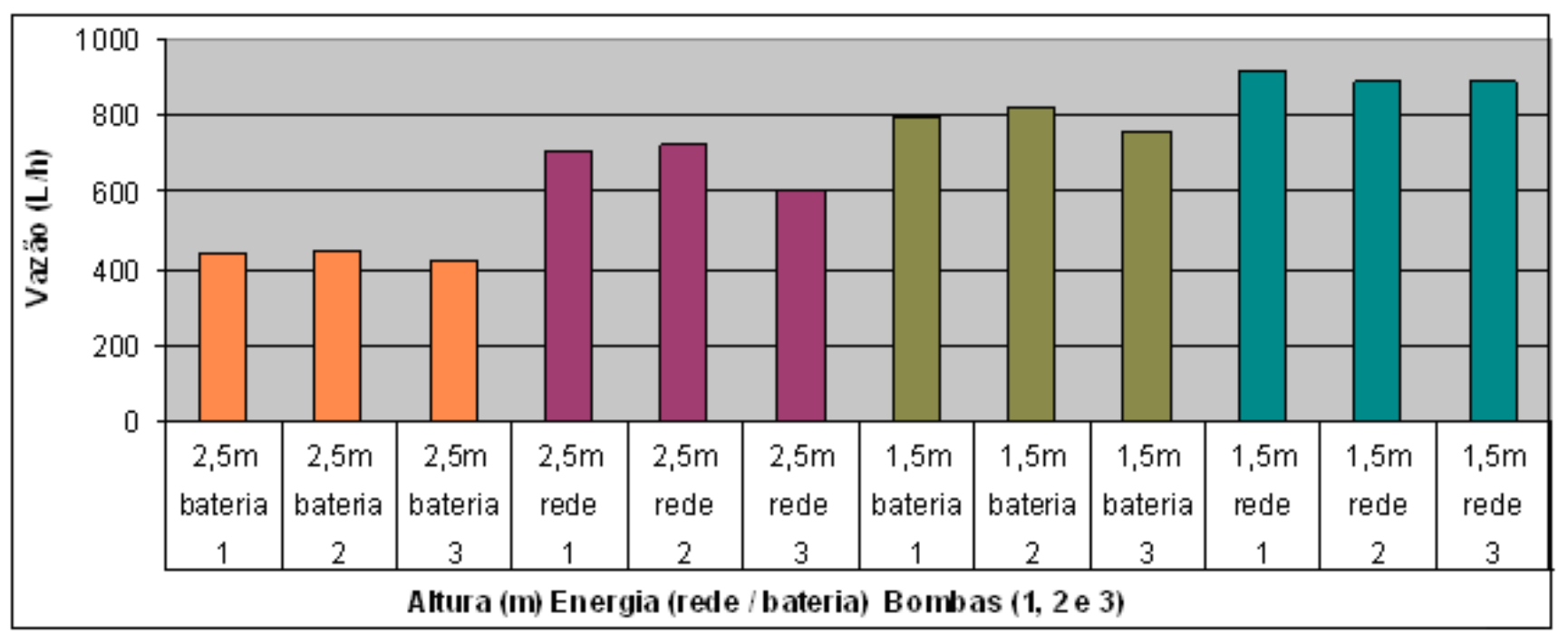

Figura 5. Desempenho de três bombas de 500 gph e 12 volts, funcionando com energia fornecida por bateria ou por rede elétrica transformada, com recalques de 1,5 e 2,5 m (performance of three 12-volt pumps of 500 gph powered by battery or by transformed electric power and elevations of 1.5 and 2.5 m). Brasília, Embrapa Hortaliças, 2008.

Utilizando a energia da bateria, o desempenho das bombas foi reduzido em relação ao fornecimento de energia elétrica transformada, lembrando que as medições foram realizadas em condições críticas de bateria usada e em meia carga, enquanto que, utilizando bateria nova, a vazão medida durante as duas primeiras horas foi de $584,5 \mathrm{~L} \mathrm{~h}^{-1}, \mathrm{o}$ que é bem mais próximo do valor 675,7 $\mathrm{L} \mathrm{h}^{-1}$, obtido com as bombas ligadas na rede elétrica, quando a altura de recalque era de $2,5 \mathrm{~m}$.

Quando a altura de recalque foi menor $(1,5 \mathrm{~m})$, o funcionamento das bombas foi mais uniforme, com pouca diferença entre a vazão obtida com acionamento por bateria (média de 791,6 L $\mathrm{h}^{-1}$ ) e o acionamento por rede elétrica transformada (média de $895,4 \mathrm{~L} \mathrm{~h}^{-1}$ ).

Para o protótipo com ar comprimido, quando foi elaborado o projeto, estimouse que a pressão a ser aplicada no reservatório seria de aproximadamente seis libras por polegada quadrada (psi), que correspondente a três metros de coluna de água (mca), o que seria suficiente para transferir a solução nutritiva de um reservatório para o outro.

Com esta estimativa, considerando que o compressor tem o pressostato regulado para funcionamento na faixa de 80 a 110 psi e supondo que o corte de energia elétrica ocorresse com a pressão mínima (80 psi); haveria então uma faixa útil de funcionamento de aproxi- madamente 74 psi, até o esgotamento do ar comprimido. Portanto, seria possível ocorrerem vários ciclos de circulação de solução nutritiva, dependendo, contudo, do volume do cilindro de ar do compressor e do volume do reservatório de solução. Poderiam ainda ser instalados vários reservatórios em série, acoplados em um único compressor.

Após a instalação do protótipo, verificou-se que a pressão mínima para funcionamento das válvulas é de $25 \mathrm{psi}$, o que reduziu em muito a eficiência do sistema em relação ao que foi previsto no projeto, conforme dados apresentados a seguir.

Quando a pressão é inferior a $25 \mathrm{psi}$, a válvula de acionamento pneumático (V2) não funciona adequadamente e ocorre o escape do ar aplicado no reservatório, não havendo a transferência da solução. Este dado não estava disponível antes da elaboração do projeto e, portanto, não havia como especificar outro modelo de válvula.

Como se trata de um protótipo construído com peças que não foram desenvolvidas para este tipo de aplicação, eles devem ser ainda melhorados.

Partindo do compressor cheio e desligado, verificou-se o número de ciclos de circulação de água, tempo de transferência do líquido entre os depósitos, volume de liquido transferido e pressão necessária para cada ciclo, obtendo-se o seguinte:
Volume por ciclo: 50,5 L (média de quatro medições)

Tempo de recalque: 2:30 minutos (média de oito medições)

Tempo de retorno: 13 minutos

Pressão exigida por ciclo: 24 libras (psi) para o compressor com cilindro de aproximadamente $45 \mathrm{~L}$

Número de ciclos por carga do compressor: 4

Consumo do compressor para encher o cilindro de ar vazio: 0,204 Kwh

Com estes resultados, considerando que a circulação de solução nutritiva nos sistemas hidropônicos é intermitente, com tempo de circulação de 15 minutos, seguido de mesmo tempo de interrupção, cada carga do compressor corresponde a uma hora de funcionamento do sistema hidropônico, sem fornecimento de energia elétrica.

Com os parâmetros obtidos é possível dimensionar os sistemas de acordo com o volume de solução a ser movimentado e com o intervalo de segurança que for conveniente, pois existem no mercado inúmeros modelos de compressores de ar, baterias automotivas de várias capacidades de carga e vários modelos de motobomba de 12 volts.

Para o sistema de ar comprimido é necessário avaliar outros modelos de válvulas solenóides, sugerindo-se utilizar as válvulas esféricas de acionamento pneumático (Guia Nei Brasil, 2009). 


\section{AGRADECIMENTOS}

Às estagiárias: Nancy dos Santos Alves, Raissa Hamanda Macedo e Débora Regina Silva, pela colaboração na montagem e coleta dos dados.

\section{REFERÊNCIAS}

CASTELLANE PD; ARAÚJO JAC. 1995 Cultivo sem solo - hidroponia. Jaboticabal: FUNEP. 43p.

COMBRINK NJJ; HARMS TM. 2001. The use of solar energy for a small recirculating hydroponic system. Acta Horticulturae.
(ISHS) n. 554, p. 285-288. http://www. actahort.org/books/554/554_30.htm

FURLANI PR. 1998. Instruções para o cultivo de hortaliças de folhas pela técnica de hidroponia NFT. Campinas, Instituto Agronômico, 1998. 30p. (IAC. Boletim Técnico, 180).

GUIA NEI BRASIL. 2009. www.nei.com.br 